\title{
Quantum dynamics of crystals of molecular nanomagnets inside a resonant cavity
}

\author{
J. Tejada, R. Amigo, and J. M. Hernandez \\ Department de Física Fonamental, Universitat de Barcelona, Diagonal 647, 08024 Barcelona, Spain \\ E. M. Chudnovsky \\ Department of Physics and Astronomy, Lehman College, City University of New York, 250 Bedford Park Boulevard West, \\ Bronx, New York 10468-1589, USA
}

(Received 21 April 2003; published 30 July 2003)

\begin{abstract}
It is found that crystals of molecular nanomagnets exhibit enhanced magnetic relaxation when placed inside a resonant cavity. A strong dependence of the magnetization curve on the geometry of the cavity has been observed, providing indirect evidence of the coherent microwave radiation by the crystals. A similar dependence has been found for a crystal placed between the Fabry-Perot superconducting mirrors.
\end{abstract}

DOI: 10.1103/PhysRevB.68.014431

PACS number(s): 75.45. $+\mathrm{j}, 75.50 . \mathrm{Xx}$

\section{INTRODUCTION}

Many fascinating magnetic effects occur at the boundary between classical and quantum physics of the angular momentum. They have been intensively investigated, both theoretically and experimentally, over the past two decades. ${ }^{1}$ The discovery of high-spin molecular nanomagnets has given a strong boost to that field. The most carefully studied nanomagnets are $\mathrm{Mn}_{12}$ and $\mathrm{Fe}_{8}$ molecular clusters. Both have spin 10 , which is 20 times the spin of electron. Such a large angular momentum is almost classical. Still crystals of $\mathrm{Mn}_{12}$ and $\mathrm{Fe}_{8}$ exhibit spectacular magnetic effects related to the quantization and quantum tunneling of the angular momentum. The present interest of physicists in these systems was ignited by the discovery of the quantum stepwise magnetic hysteresis in $\mathrm{Mn}_{12}$ acetate. ${ }^{2}$ Steps of quantum nature in the magnetization curve of magnetic clusters and single ions have been seen in the past. ${ }^{3}$ However, in $\mathrm{Mn}_{12}$ acetate the steps are more dramatic due to the high value of spin and long lifetimes of metastable spin states. The explanation of the magnetization curve of $\mathrm{Mn}_{12}$-acetate crystals is based upon the structure of the spin energy levels of $\mathrm{Mn}_{12}$ molecules.

The $\mathrm{Mn}_{12}$ cluster has a tetragonal symmetry. ${ }^{4}$ In the magnetic field $H_{z}$, applied along the tetragonal axis, it is described by the Hamiltonian

$$
\mathcal{H}=-D S_{z}^{2}-A S_{z}^{4}-g \mu_{B} S_{z} H_{z}+\mathcal{H}^{\prime},
$$

where $S=10, D \approx 0.55 \mathrm{~K}, A \approx 1.2 \times 10^{-3} \mathrm{~K}, g \approx 1.94$, and $\mathcal{H}^{\prime}$ contains small terms that do not commute with $S_{z}$. If one neglects $\mathcal{H}^{\prime}$, then, in a zero field, the ground state of the cluster is double degenerate. The dependence of the energy of the $\mathrm{Mn}_{12}$ cluster on the magnetic quantum number $m$ at $H_{z}=0$ is shown by the inverted parabola in Fig. 1. The two energy minima $m= \pm 10$ correspond to the spin looking up or down along the $Z$ axis. They are separated by the magnetic anisotropy barrier $U \approx 65 \mathrm{~K}$. At a nonzero field applied in the positive $Z$ direction, the negative $m$ levels in Fig. 1 move up while the positive $m$ levels move down. The trivial algebra of Eq. (1) yields that the $m$ and $m^{\prime}$ eigenvalues of $S_{z}$ come to resonance at

$$
H_{z}=H_{m m^{\prime}}=-\left(m+m^{\prime}\right) \frac{D}{g \mu_{B}}\left[1+\frac{A}{D}\left(m^{2}+m^{\prime 2}\right)\right],
$$

see Fig. 1. At $H_{z} \neq H_{m m^{\prime}}$ transitions between negative and positive $m$ occur due to the thermal activation over the anisotropy barrier (upward arrows in Fig. 1). However, at $H_{z}$ $=H_{m m^{\prime}}$ the transitions are combinations of thermal activation and quantum tunneling ${ }^{5-8}$ (horizontal arrows in Fig. 1). Thus, for $H_{z}=H_{m m}$ the anisotropy barrier is effectively reduced by tunneling and the magnetic relaxation towards the direction of the field is faster than at $H_{z} \neq H_{m m^{\prime}}$. This picture, first established by magnetic measurements, ${ }^{2}$ has been confirmed by the careful EPR study of spin levels in $\mathrm{Mn}_{12}$-acetate. ${ }^{9-15}$

The EPR experiments have demonstrated noticeable resonant absorption of electromagnetic radiation by molecular magnets. Most recently, it has been suggested that a crystal

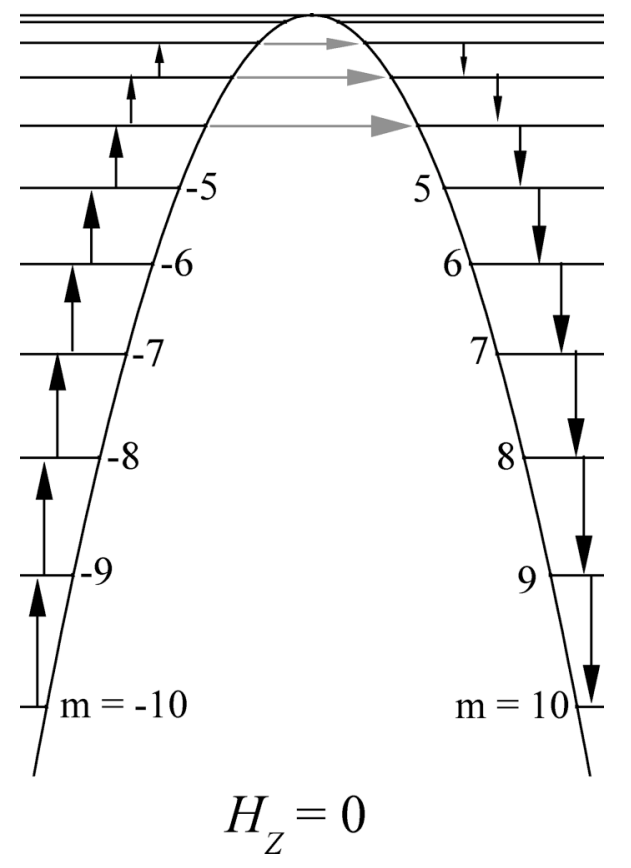

FIG. 1. Thermally assisted spin tunneling in spin-10 molecular magnets. 


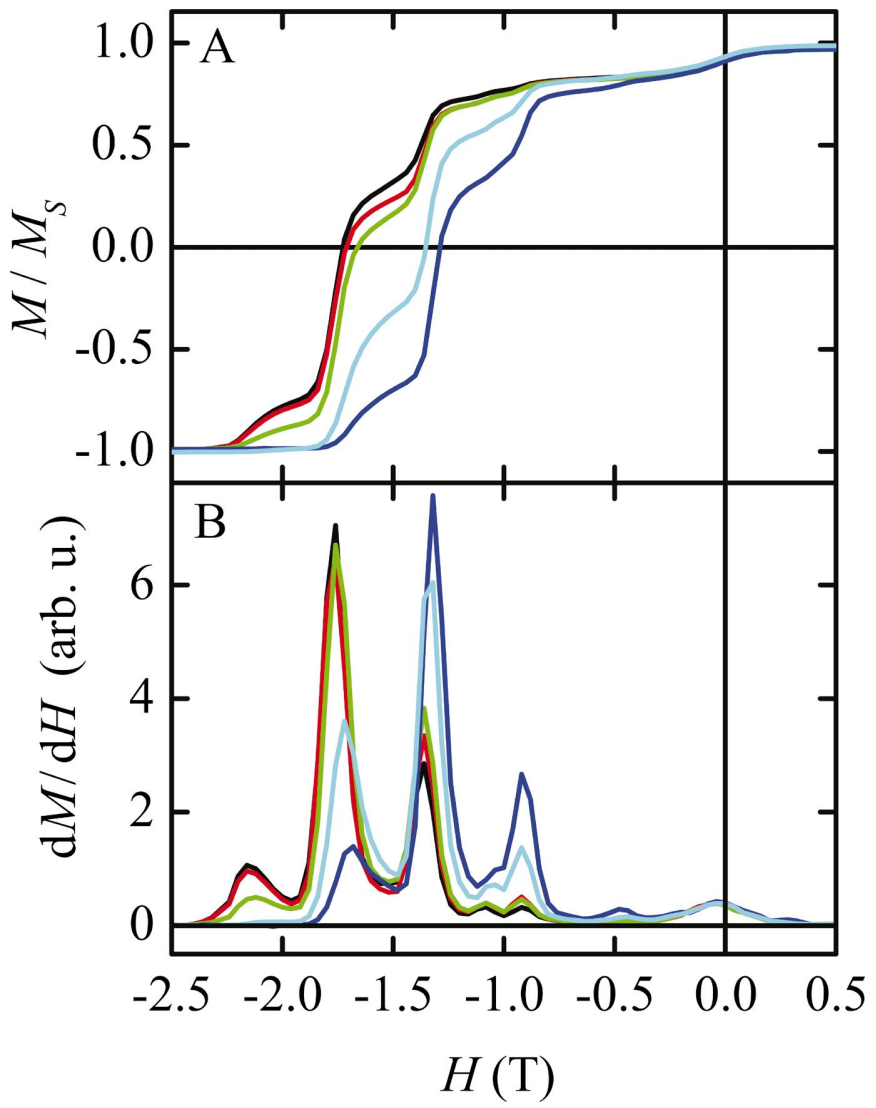

FIG. 2. (Color) (a) Demagnetization curves, $M(H)$, of $\mathrm{Mn}_{12}$-acetate crystal at $T=2.0 \mathrm{~K}$ inside the resonant cavity of diameter $1.6 \mathrm{~mm}$ for five different lengths of the cavity: $L$ $=21.0 \mathrm{~mm}$ (black), $L=20.9 \mathrm{~mm}$ (red), $L=20.1 \mathrm{~mm}$ (green), $L$ $=19.8 \mathrm{~mm}$ (dark blue), and $L=19.5 \mathrm{~mm}$ (light blue); (b) $d M / d H$ for the curves shown in (a).

of magnetic molecules can also be a powerful source of coherent microwave radiation. ${ }^{16}$ The authors of Ref. 16 have studied a special kind the super-radiance arising due to the level crossing. The effect arises from the fact that the wavelength of photons emitted in transitions between the spin levels of molecular magnets is typically large compared to the size of the crystal, so that the radiation transition becomes a collective action by a macroscopic number of molecules. On general grounds, it is clear that the maser action may also arise from effects other than the level crossing. Indeed, by simply placing the crystal in the magnetic field, one can easily create the inverted population of the spin energy levels. The radiation can, then, be produced in a laser fashion due to the electromagnetic relaxation of the crystal towards the minimum of the magnetic energy (downward arrows in Fig. 1). Such effect, if observed, would open the possibility of building a microwave maser pumped by the magnetic field. In this paper we first provide an indirect experimental support to this idea by demonstrating a strong interaction between the $\mathrm{Mn}_{12}$ crystal and a microwave cavity. After presenting the data we will discuss why such a demonstration may be the evidence of collective radiation effects.

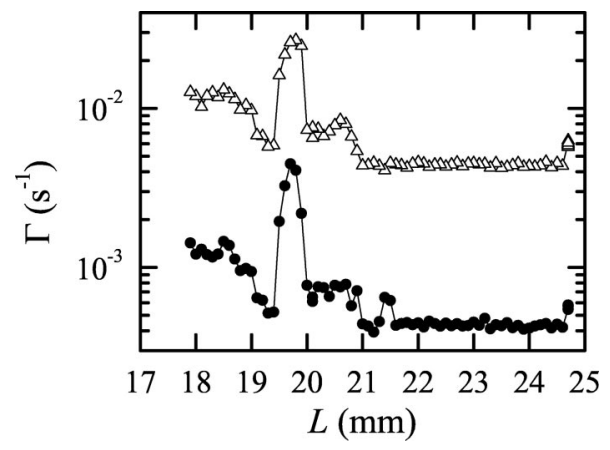

FIG. 3. The rate of magnetic relaxation in a $\mathrm{Mn}_{12}$-acetate crystal inside the cavity of diameter $1.6 \mathrm{~mm}$ at $T=2.0 \mathrm{~K}$ as a function of the cavity length at the second (solid circles) and third (open triangles) resonance fields. The error bars are of the size of the points.

\section{EXPERIMENTS}

The purpose of our experiments was to test whether the placing of the crystal inside a resonant cavity would result in

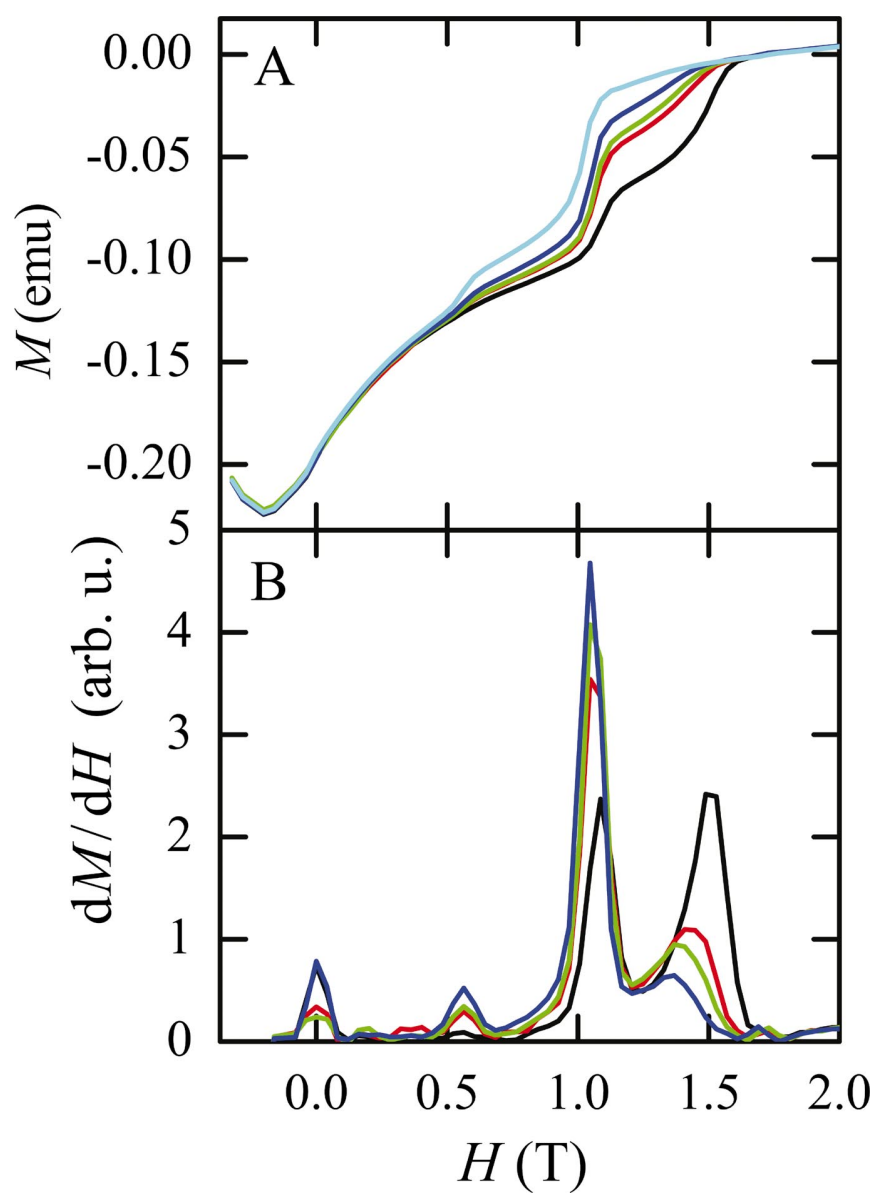

FIG. 4. (Color) (a) Demagnetization curves, $M(H)$, of the Fabry-Perot setup at $T=2.0 \mathrm{~K}$ for five distances between superconducting mirrors: $d=4.3 \mathrm{~mm}$ (black), $d=4.7 \mathrm{~mm} \quad$ (red), $d=5.1 \mathrm{~mm}$ (green), $d=5.3 \mathrm{~mm}$ (dark blue), and $d=6.0 \mathrm{~mm}$ (light blue); (b) $d M / d H$ calculated from the demagnetization curves in (a) after subtracting the contribution of the superconductors. 
any change in the magnetization curve. Single crystals of $\mathrm{Mn}_{12}$ acetate have been grown with the average length and diameter of about $2 \mathrm{~mm}$, and $0.2 \mathrm{~mm}$, respectively. The elongation of the crystals was along the $c$ axis. The conventional composition and the structure of the crystals ${ }^{17}$ have been established by chemical, infrared, and x-ray diffraction techniques. In addition, the dc and ac magnetometry of the crystals have been performed. The same values of the blocking temperature and resonance fields, as previously reported, ${ }^{2,18,19}$ have been found. In constructing resonant cavities we followed the procedures described in Refs. 20 and 21. Five cylindrical cavities of different diameter and adjustable length were constructed using $99.99 \%$ purity copper. Two diameters were used: $1.6 \mathrm{~mm}$ and $3.2 \mathrm{~mm}$. The length of the cavity was controlled with the help of the samepurity copper rod connected to the upper surface of the cavity. The micrometric stepping motor control system was used that had the spatial resolution of $1 \mu \mathrm{m}$. The inner lateral and planar surfaces of the cavity were polished to a roughness of less than $10 \mu \mathrm{m}$ to achieve the quality factor between $Q$ $=10^{3}$ and $Q=10^{4}$. The crystals were fixed in a vertical position at the bottom of the cavities, with the $c$ axis of the crystal parallel to the cylindrical axis of the cavity. The grease of high thermal conductivity was used to attach the crystal to the bottom of the cavity. The cavity containing the crystal was immersed in a helium gas inside the MPMS SQUID magnetometer. The upper moving surface made the cavity nonhermetic, thus allowing the exchange of helium with the outside reservoir. The temperature inside and outside the cavity was monitored by carbon thermometers.

Before placing crystals inside the cavities the magnetic signals from the crystals and from the cavities have been measured independently. The signal from the crystal was always two orders of magnitude greater than the paramagnetic signal from the cavity. We then proceeded to the measurements of crystals inside the cavities. The system was first saturated by a $5 \mathrm{~T}$ field applied along the $c$ axis of the crystal. Then the field was swept in the opposite direction at the rate $r=40 \mathrm{Oe} / \mathrm{s}$. A very similar behavior has been found in all the cavities. Typical demagnetization curves from $\mathrm{Mn}_{12}$-acetate crystals inside a resonant cavity are shown in Fig. 2. The dependence of the relaxation rate, $\quad \Gamma=r \mid M(H)$ $-\left.M_{e q}(H)\right|^{-1}(d M / d H)$ (with $M_{e q}$ being equilibrium magnetization), on the length of the cavity at two resonance fields is shown in Fig. 3.

In a separate set of experiments we placed the $\mathrm{Mn}_{12}$-acetate crystal between two Fabry-Perot superconducting mirrors. The mirrors were prepared using method described in Ref. 22. The 200-nm YBaCuO layers were deposited by pulsed laser deposition on a $1-\mu \mathrm{m} \mathrm{SrTiO}_{3}$ substrate. Their superconducting properties below $90 \mathrm{~K}$ were verified by magnetic measurements. The diamagnetic signal from the mirrors was found to be comparable to the magnitude of the signal from the crystal, but independent the distance between the mirrors. The demagnetization curves from the FabryPerot setup are shown in Fig. 4. The diamagnetic signal from the superconductors was subtracted from the total signal to obtain the curve of Fig. 4(b). The dependence of $d M / d H$ on the distance between the mirrors at $H_{z}=0$ is shown in Fig. 5 .

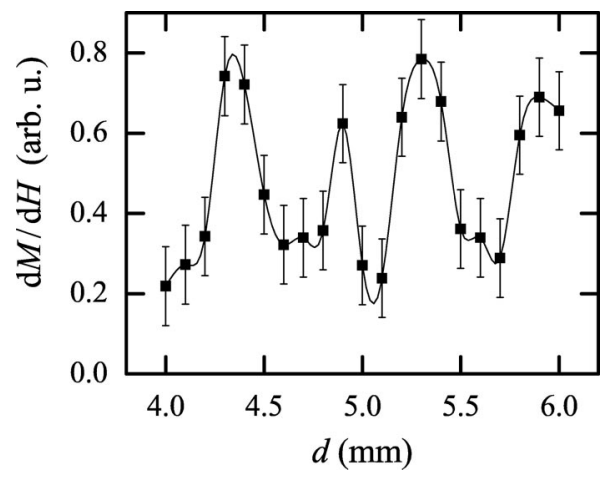

FIG. 5. $d M / d H$ at $H_{z}=0$ and $T=2.0 \mathrm{~K}$ for a $\mathrm{Mn}_{12}$-acetate crystal between superconducting mirrors as a function of the distance between the mirrors.

The experimental data clearly demonstrate the dependence of the magnetic relaxation on the geometry of the cavity. We have verified that this phenomenon appears only in cavities of high quality factor. The temperature variation was found not to exceed $0.3 \%$. Such small fluctuations of temperature cannot account for any measurable change in the relaxation rate. All data on the dependence of the rate on the length of the cavity and the magnetic field were reproducible within a $6 \%$ experimental error. Similar effects have been observed in a $\mathrm{Mn}_{12}$-acetate crystal placed between superconducting mirrors, that is, when the helium gas circulated freely around the crystal. We, therefore, concluded that the observed phenomenon had nothing to do with thermal effects and was, in fact, due to the microwave properties of the cavity.

\section{DISCUSSION}

We shall now discuss why the detection of the above effect may constitute the evidence of the coherent radiation inside the cavity. Consider a crystal of $\mathrm{Mn}_{12}$ acetate magnetized in the negative $Z$ direction. At $H \ll 10 \mathrm{~T}$ the distance $E_{1}$ between $m=-10$ and $m=-9$ levels in Fig. 1 is about $14 \mathrm{~K}$. Thus, at $T \ll E_{1}$, most of the molecules occupy the $m=-10$ level. The occupation numbers of the excited states scale as $\exp (-E / T)$, where $E$ is the energy distance from $m$ $=-10$. In the absence of a very large transverse field, quantum tunneling from $m=-10$ in $\mathrm{Mn}_{12}$ has a negligibly low probability. The thermodynamic equilibrium is achieved either through thermal activation or through thermally assisted quantum tunneling from excited states. The activation of molecules to the excited states is believed to be due to the absorption of phonons. At $T \ll E_{1}$ the rate of phonon-induced transitions from $m=-10$ to $m=-9$ for $\mathrm{Mn}_{12}$-acetate is given by ${ }^{23}$

$$
\Gamma_{\text {phonon }}=\frac{\hbar S \omega_{1}^{5}}{12 \pi \rho c_{s}^{5}} \exp \left(-\frac{\hbar \omega_{1}}{T}\right)
$$

where $\omega_{1}=E_{1} / \hbar$ is the frequency of the phonon $\left(f_{1}\right.$ $\left.=\omega_{1} / 2 \pi \approx 300 \mathrm{GHz}\right), \rho \sim 1.8 \mathrm{~g} / \mathrm{cm}^{3}$ is the mass density of 
the crystal, and $c_{s} \sim 10^{5} \mathrm{~cm} / \mathrm{s}$ is the speed of the transverse sound. At $2 \mathrm{~K}$, Eq. (3) yields $\Gamma_{\text {phonon }} \sim 5 \times 10^{5} \mathrm{~s}^{-1}$.

In order to have any effect due to the electromagnetic radiation, the absorption of photons must have a rate comparable to the rate of the absorption of phonons. This can be achieved, e.g., in the EPR experiment in which the sample is placed in the ac magnetic field $H_{a c}$ oscillating at frequency $f_{1}$. The rate of the absorption of photons in the EPR setup is given by ${ }^{24}$

$$
\Gamma_{\text {photon }}=k \frac{S g^{2} \mu_{B}^{2}}{\hbar^{2}} H_{a c}^{2} F(\omega),
$$

where $k$ is a numerical factor of order 1 that depends on the polarization of photons and $F(\omega)$ is the shape function of the resonance. In the case of, e.g., the Lorentzian line of width $\Delta \omega$, the shape function is given by

$$
F(\omega)=\frac{1}{\pi} \frac{\Delta \omega}{(\Delta \omega)^{2}+\left(\omega-\omega_{1}\right)^{2}} .
$$

The maximal EPR rate, $\Gamma_{\text {photon }} \sim g \mu_{B} S H_{a c}^{2} / \hbar \Delta H$, is achieved at $\omega=\omega_{1}$, where $\Delta H$ is the field width of the line, $\hbar \Delta \omega=g \mu_{B} \Delta H$. In $\mathrm{Mn}_{12}, \Delta H$ is of order of 400 Oe. Consequently, the rate of the absorption of EPR photons becomes comparable to the phonon rate of Eq. (3) at $H_{a c} \sim 1$ Oe.

In our experiments no external ac magnetic field has been used. The crystal of $\mathrm{Mn}_{12}$ acetate was simply placed inside a resonant cavity and the magnetization curve has been measured. Let us assume for the moment that only thermal photons are available for the transitions between, e.g., $m=-10$ and $m=-9$ levels. At $2 \mathrm{~K}$ their magnetic field is of order $3 \times 10^{-8} \mathrm{Oe}$ as compared to the $1 \mathrm{Oe}$ ac field needed to beat the phonon rate in the EPR experiment. Thus, thermal photons inside the cavity excite $\mathrm{Mn}_{12}$ molecules at a rate that is 15 orders of magnitude lower than the phonon rate. This is mainly due to the fact that at any temperature each cubic centimeter of a solid contains $\left(c / c_{s}\right)^{3} \sim 10^{15}$ times more thermal phonons than thermal photons. At $T=2 \mathrm{~K}$ our cavity would have $10^{-3}$ average number of thermal photons of energy $E_{1}$. Consequently, the cavity should play absolutely no role in the magnetic relaxation unless it occasionally acquires a very large number of nonthermal photons. There is only one source of such photons, it is the crystal itself. To change the orientation of the magnetic moment, the $\mathrm{Mn}_{12}$ molecule must first go up the staircase of spin levels and then go down (see Fig. 1). Thus, the magnetic relaxation in the crystal creates massive inverse population of the energy levels. The shortest wavelength of a photon corresponds to the transition from $m=9$ to $m=10$. For $\mathrm{Mn}_{12}$ at $H=0$ it is about $1 \mathrm{~mm}$. Consequently, for a crystal of size $2 \mathrm{~mm}$, the phase of the emitted photons is the same for a macroscopically large number of molecules, $N_{S R}$. In that case, the emission of photons by different molecules becomes correlated and a burst of the super-radiance may occur. ${ }^{16,25}$ This effect is much stronger for photons than for phonons because the wavelength of photons is $\left(c / c_{s}\right)$ times the wavelength of phonons of the same energy. Thus, the $\left(c_{s} / c\right)^{3}$ smallness of the phase space of photons in comparison with the phase space of phonons is compensated by the $\left(c / c_{s}\right)^{3}$ times greater $N_{S R}$ for photons as compared to phonons.

The effect of a macroscopically large number of coherent photons inside the cavity is equivalent to the effect of the ac magnetic field. The reabsorption of the emitted photons increases the population of the levels with $m>-10$ as compared to their thermal population. Contrary to the EPR experiment, however, in our case the photons absorbed in the left well in Fig. 1 must be the same photons that are emitted in the right well. Obviously, the super-radiant photons cannot be present in the cavity during the entire $40 \mathrm{Oe} / \mathrm{s}$ sweep across the 400-Oe wide resonance. Many short bursts of super-radiance, each resulting in the simultaneous spin reversal in a small volume, are more likely. It has been demonstrated $^{16}$ that the maximal super-radiance rate can exceed the spin-phonon relaxation rate by a factor as large as $10^{4}$, making the effect still possible in a $10^{-4}$ fraction of a millimeter-size sample. To affect the magnetic relaxation, one of the frequencies $\omega$ of the emitted photons should satisfy two conditions. First, $\hbar \omega$ must coincide with one of the distances between levels in the metastable well (the left staircase in Fig. 1). It occurs for certain values of the field uniquely determined by the spin Hamiltonian. Second, $\omega$ must coincide with one of the resonances of the cavity. It is achieved by manipulating the length of the cylindrical cavity or the distance between the superconducting mirrors. A strong support to this picture comes from the fact that the main period of oscillations of the relaxation rate on the distance between the superconducting mirrors, (see Fig. 5) is about $0.5 \mathrm{~mm}$, which is one-half of the wavelength of the 300-GHz photons responsible for the transitions between $m$ $=-10$ and $m=-9$ levels. This is the most important resonance because almost all molecules initially occupy the $m$ $=-10$ state. Exciting these molecules to the $m=-9$ level alone changes the effective energy barrier by $E_{1} \approx 14 \mathrm{~K}$, thus increasing the relaxation rate by a factor $\exp \left(E_{1} / T\right) \sim 10^{3}$. Notice that for cavities, the length dependence of the relaxation rate is more complicated. Photons emitted in the magnetic dipole transitions should be in resonance with the TM modes of the cavity. The latter, for a cavity of radius $R$ and length $L$, satisfy

$$
\omega_{m n p}^{2}=\frac{c^{2} \kappa_{m n}^{2}}{R^{2}}+\frac{\pi^{2} c^{2} p^{2}}{L^{2}},
$$

where $p=1,2,3, \ldots$ and $x=\kappa_{m n}$ is the nth zero of the Bessel function $J_{m}(x)$. Matching the spectrum of the spin levels of $\mathrm{Mn}_{12}$ acetate with the spectrum of the cavity should be the way to explain the dependence of the magnetic relaxation on the length of the cavity. We have not succeeded in that task so far because of the uncertainties in the rather dense spectra of the cavities and the crystals.

The coherent effects discussed in this paper favor narrow spin levels. In the absence of crystal disorder, this require a uniformly magnetized sample, which can be achieved by choosing very alongated crystals of the kind used in our experiments. Besides dipolar fields, the broadening of the levels may also come from chemical disorder, various de- 
fects of the crystal structure ${ }^{26}$ and from hyperfine interactions of molecular spins with randomly oriented nuclear spins. ${ }^{27}$ These effects should be weaker in $\mathrm{Fe}_{8}$ than in $\mathrm{Mn}_{12}$, making $\mathrm{Fe}_{8}$ a more attractive candidate for the study of coherent electromagnetic effects. However, the temperature needed to discard thermal transitions in $\mathrm{Fe}_{8}$ is lower than for $\mathrm{Mn}_{12}$ and is below the capabilities of the MPMS SQUID magnetometer used in our experiments. This was the primary reason why we chose $\mathrm{Mn}_{12}$. Despite the fact that it appears to be a rather disordered system, recently a few groups have reported experimental evidence $\mathrm{e}^{28-30}$ of the discrete nature of the disorder in $\mathrm{Mn}_{12}$. In effect, the disorder seems to stem from a finite number of chemical isomers of $\mathrm{Mn}_{12}$ acetate. This would result in a small number of families of narrow levels that would allow coherent microwave absorption and radiation.

\section{CONCLUSIONS}

In conclusion, we have found that the magnetic relaxation of $\mathrm{Mn}_{12}$-acetate crystals inside a resonant cavity differs from the magnetic relaxation outside the cavity. It seems impossible to understand this effect without invoking the superradiance. Of course, the unambiguous proof of the collective electromagnetic effects in crystals of molecular nanomagnets may only come from direct measurements of the microwave radiation.

\section{ACKNOWLEDGMENTS}

We thank F. Sánchez and M. Varela for their help in preparing the Fabry-Perot superconducting mirrors. The financial support from the EU is gratefully acknowledged. The work of E.M.C. has been supported by the U.S. NSF Grant No. 9978882.
${ }^{1}$ E. M. Chudnovsky and J. Tejada, Macroscopic Quantum Tunneling of the Magnetic Moment (Cambridge University Press, Cambridge, England, 1998).

${ }^{2}$ J.R. Friedman, M.P. Sarachik, J. Tejada, and R. Ziolo, Phys. Rev. Lett. 76, 3830 (1996).

${ }^{3}$ See for review Y. Shapira, and V. Bindilatti, J. Appl. Phys. 92, 4155 (2002).

${ }^{4}$ R. Sessoli, D. Gatteschi, A. Caneschi, and M.A. Novak, Nature (London) 365, 141 (1993).

${ }^{5}$ J.L. van Hemmen and A. Sütő, Europhys. Lett. 1, 481 (1986); Physica B \& C 141B, 37 (1986); G. Sharf, W.F. Wreszinski, and J.L. van Hemmen, J. Phys. A 20, 4309 (1987).

${ }^{6}$ M. Enz and R. Schilling, J. Phys. C 19, L711 (1986).

${ }^{7}$ E.M. Chudnovsky, Zh. Éksp. Teor. Fiz. 77, 2158 (1979) [Sov. Phys. JETP 50, 1035 (1979)]; E.M. Chudnovsky and L. Gunther, Phys. Rev. Lett. 60, 661 (1988).

${ }^{8}$ D.A. Garanin, J. Phys. A 24, L61 (1991).

${ }^{9}$ A.L. Barra, D. Gatteschi, and R. Sessoli, Phys. Rev. B 56, 8192 (1997)

${ }^{10}$ S. Hill, J.A.A.J. Perenboom, N.S. Dalal, T. Hathaway, T. Stalcup, and J.S. Brooks, Phys. Rev. Lett. 80, 2453 (1998).

${ }^{11}$ E. del Barco, J.M. Hernandez, J. Tejada, N. Biskup, R. Achey, I. Rutel, N. Dalal, and J. Brooks, Phys. Rev. B 62, 3018 (2000).

${ }^{12}$ A.B. Sushkov, B.R. Jones, J.L. Musfeldt, Y.J. Wang, R.M. Achey, and N.S. Dalal, Phys. Rev. B 63, 214408 (2001).

${ }^{13}$ B. Parks, J. Loomis, E. Rumberger, D.N. Hendrickson, and G. Christou, Phys. Rev. B 64, 184426 (2001).

${ }^{14}$ K. Park, M.A. Novotny, N.S. Dalal, S. Hill, and P.A. Rikvold, J. Appl. Phys. 91, 7167 (2002).

${ }^{15}$ M. Dressel, B. Gorshunov, K. Rajagopal, S. Vongtragool, and A. A. Mukhin, Phys. Rev. B 67, 060405 (2003).

${ }^{16}$ E.M. Chudnovsky and D.A. Garanin, Phys. Rev. Lett. 89, 157201 (2002).

${ }^{17}$ T. Lis, Acta Crystallogr., Sect. B: Struct. Crystallogr. Cryst.
Chem. B36, 2042 (1980).

${ }^{18}$ J.M. Hernandez, X.X. Zhang, F. Luis, J. Bartolomé, J. Tejada, and R. Ziolo, Europhys. Lett. 35, 301 (1996).

${ }^{19}$ L. Thomas, F. Lionti, R. Ballou, D. Gatteschi, R. Sessoli, and B. Barbara, Nature (London) 383, 145 (1996).

${ }^{20}$ M. Mola, S. Hill, P. Goy, and M. Gross, Rev. Sci. Instrum. 71, 186 (2000).

${ }^{21}$ O. Klein, S. Donovan, M. Dressel, and G. Grüner, Int. J. Infrared Millim. Waves 14, 2423 (1993); S. Donovan, O. Klein, M. Dressel, K. Holezer, G. Grüner, ibid. 14, 2459 (1993); M. Dressel, O. Klein, S. Donovan, and G. Grüner, ibid. 14, 2489 (1993).

${ }^{22}$ A. Garcia-Santiago, F. Sánchez, M. Varela, and J. Tejada, Appl. Phys. Lett. 77, 2900 (2000).

${ }^{23}$ D.A. Garanin and E.M. Chudnovsky, Phys. Rev. B 56, 11102 (1997); E.M. Chudnovsky and X. Martinez-Hidalgo, ibid. 66, 054412 (2002).

${ }^{24}$ A. Abragam and B. Bleaney, Electron Paramagnetic Resonance of Transition Ions (Clarendon Press, Oxford, England, 1970).

${ }^{25}$ See M. Gross and S. Haroche, Phys. Rep. 93, 301 (1982); L.I. Menshikov, Usp. Fiz. Nauk 169, 113 (1999) [Sov. Phys. Usp. 42, 107 (1999)].

${ }^{26}$ E.M. Chudnovsky and D.A. Garanin, Phys. Rev. Lett. 87, 187203 (2001); D.A. Garanin and E.M. Chudnovsky, Phys. Rev. B 65, 094423 (2002).

${ }^{27}$ D.A. Garanin, E.M. Chudnovsky, and R. Schilling, Phys. Rev. B 61, 12204 (2000).

${ }^{28}$ A. Cornia, R. Sessoli, L. Sorace, D. Gatteschi, A.L. Barra, and C. Daiguebonne, Phys. Rev. Lett. 89, 257201 (2002).

${ }^{29}$ S. Hill, R. S. Edwards, S. I. Jones, N. S. Dalal, and J. M. North, Phys. Rev. Lett. 90, 217204 (2003).

${ }^{30}$ E. del Barco, A.D. Kent, E.M. Rumberger, D.N. Hendrickson, and G. Christou, cond-mat/0304206, Phys. Rev. Lett. (to be published). 